\title{
AETIOLOGICAL AND CLINICAL PROFILE OF HYPERTENSIVE EMERGENCIES IN A TERTIARY CARE HOSPITAL
}

\author{
Ashhar C1, Mohamed Shaan²
}

1 Junior Resident, Department of Emergency Medicine, Government Medical College, Kozhikode, Kerala.

${ }^{2}$ Associate Professor, Department of General Medicine, Government Medical College, Manjeri, Kerala.

\section{BACKGROUND}

ABSTRACT

The aetiological and clinical profile of patients with hypertensive emergencies presenting to hospitals in our country is poorly known. Systemic Hypertension is a common medical condition affecting over 1 billion people worldwide. Epidemiological studies indicate that the prevalence of hypertension in adults is around $15 \%$ to $22 \%$, depending on the population considered. Although, chronic hypertension was an established risk factor for cardiovascular, cerebrovascular and renal disease, acute elevations in blood pressure can result in acute end organ damage with significant morbidity.

The objective of the present study was to list the probable aetiologies, clinical profile, target organ damage spectrum and mortality pattern and its association with variables.

\section{MATERIALS AND METHODS}

The present study was done in patients admitted at Calicut Medical College Hospital, a tertiary referral centre situated in Northern Kerala, over a period of one year. Study design was descriptive study. The study population included patients admitted to emergency department with severely elevated BP with clinical or laboratory evidence of acute organ damage. The clinical and laboratory profile and mortality pattern and its association with variables were evaluated and analysed.

\section{RESULTS}

The mean age was found to be 61 years with a standard deviation of 12 years. Predominant presenting symptoms were acute coronary syndrome followed by intracerebral haemorrhage. Depressed sensorium was present in patients and is significantly associated with mortality. Mean systolic blood pressure was $200 \mathrm{mmHg}$ and mean diastolic blood pressure was $124 \mathrm{mmHg}$. High systolic and diastolic blood pressure were significantly associated with mortality (p values- 0.00 and 0.00 ). Serum creatinine was above $2 \mathrm{mg} / \mathrm{dL}$ in 40 patients and high creatinine value was statistically related with mortality. End organ damages were acute coronary syndrome followed by intracerebral haemorrhage. The in-hospital mortality was $28 \%$.

\section{CONCLUSION}

Males had a higher chance of developing hypertensive emergencies. The most common target of organ damage was acute coronary syndrome. Higher systolic and diastolic blood pressure were highly associated with mortality.

Limitations- The smaller size of the study limits the ability to offer firm conclusions about the relationship between hypertensive emergency and aetiological factors.

\section{KEY WORDS}

Arterial Hypertension; Hypertensive Emergencies; Hypertensive End Organ Damage.

HOW TO CITE THIS ARTICLE: Ashhar C, Shaan M. Aetiological and clinical profile of hypertensive emergencies in a tertiary care hospital. J. Evolution Med. Dent. Sci. 2018;7(20):2491-2497, DOI: 10.14260/jemds/2018/560

\section{BACKGROUND \\ Systemic Hypertension is a common medical condition} affecting over 1 billion people worldwide. ${ }^{1}$ Epidemiological studies indicate that the prevalence of hypertension in adults is around $15 \%$ to $22 \%$ depending on the population considered. $^{2}$ Although, chronic hypertension was an established risk factor for cardiovascular, cerebrovascular and renal disease, acute elevations in blood pressure can result in acute end organ damage with significant morbidity. ${ }^{3}$ Although, great strides have been made in the treatment of

'Financial or Other Competing Interest': None.

Submission 03-04-2018, Peer Review 27-04-2018,

Acceptance 03-05-2018, Published 14-05-2018.

Corresponding Author:

Dr. Mohamed Shaan,

Associate Professor,

Department of General Medicine,

Government Medical College, Manjeri,

MASKAN, 5/1986 B, KP Chandran Road,

PO Puthiyara, Calicut-673004, Kerala.

E-mail: shaanmohamed77@gmail.com DOI: $10.14260 /$ jemds $/ 2018 / 560$ hypertension which accounts for more than one-fourth of all medical urgencies and emergencies, patients still present in hypertensive crisis.

A hypertensive emergency is characterised by rapid deterioration of target-organs and poses an immediate threat to life. Due to the association of hypertensive emergencies with various cerebral, cardiac and renal complications there is an urgent need to recognise this condition, so as to reduce the burden associated with it in terms of increased morbidity and mortality in the society.

This study provides a view on hypertensive emergencies, focusing on one of its most serious complications: hypertensive emergency, a serious condition in which blood pressure undergoes an elevation $>180 / 120 \mathrm{mmHg}$ and is associated with end organ dysfunction (e.g. heart, kidneys, eyes or brain). There are only a few studies in our country in the field of hypertensive emergency and only a few studies compare the mortality determinants. Hence, we undertake the present study to prospectively find the relationship between aetiological profile and clinical profile with mortality 
and thereby trying to predict the prognosis of the type of end organ damages.

\section{Objectives}

1. To list the probable aetiology of the patients with hypertensive emergencies.

2. To enlist the clinical profile and spectrum of target organ damage in patients with hypertensive emergencies.

3. To measure the mortality pattern of hypertensive emergencies and its statistical significance with various factors.

\section{MATERIALS AND METHODS}

The present study was done in Calicut Medical College Hospital, a tertiary referral centre situated in Northern Kerala over a period of one year.

\section{Study Design}

Descriptive study.

\section{Study Setting}

Emergency Department of Calicut Medical College, a tertiary care centre in Northern Kerala.

\section{Study Period}

August 2015 - July 2016, one year.

\section{Study Subjects}

All patients presenting with hypertensive emergencies at Calicut Medical College, Emergency Department during the study period.

\section{Selection Criteria/ Inclusion Criteria}

1. Patients above 18 years of age.

2. Systolic blood pressure $\geq 180 \mathrm{mmHg}$ and diastolic blood pressure $\geq 120 \mathrm{mmHg}$.

3. Evidence of end organ damage, either clinically or laboratory findings.

\section{Sampling Method}

Selecting patients fulfilling the criteria consecutively during the data collection period. Data collection period is done in 4 months.

\section{Sample Size}

No exact prevalence for hypertensive emergencies were recorded in India. Around 600 patients with hypertensive emergencies attend to Emergency Department, Calicut Medical College per year. In view of shorter duration of data collection, sample size was taken as 150 patients for convenience.

\section{Study Tool}

Semi-Structured Questionnaire.

Data were double entered into Microsoft Excel and cleaned. Analysis was performed using SPSS software version 18. Results were described using proportions (\%) for categorical data and mean \pm SD for quantitative variables. P values $<0.05$ were considered as significant predictors.

\section{RESULTS}

Socio-Demographic Profile

Age

The mean age in the present study group was found to be 61.34 years. The age group ranges from 26 to 90 years. Most of the hypertensive emergency cases presented in the $7^{\text {th }}$ decade. 112 patients were males.

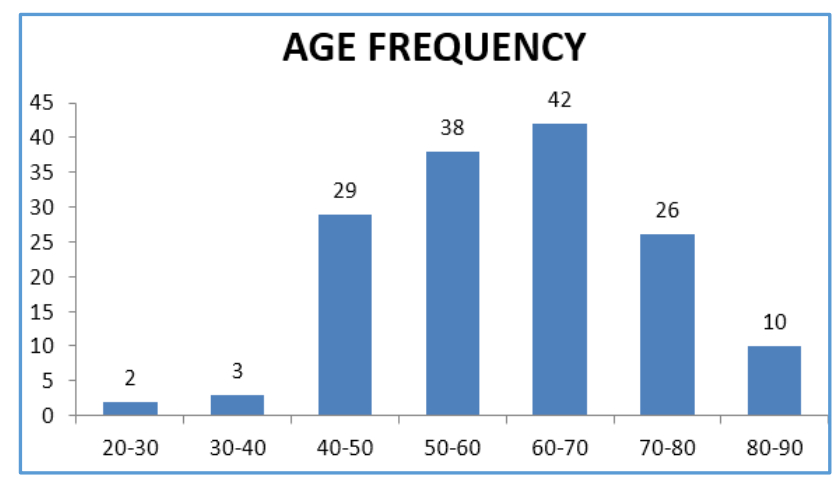

Figure 1. The Decade Wise distribution of Age

\section{Aetiological Profile \\ Lifestyle \\ 79 patients had good physical activity (52.6\%) and 71 patients had sedentary lifestyle (47.3\%).}

\section{Hypertensive Status}

109 patients had history of hypertension (72.6\%). The hypertensive status of rest of the patients were not known. Out of 109 hypertensives, only 32 patients were taking regular antihypertensive treatment (29.73\%).

\section{Diabetes Mellitus}

54 patients were known diabetics (36\%). Out of 54 patients, 19 patients were taking regular medications (35\%).

\section{Smoking}

90 patients were smokers $(60 \%)$.

\section{Alcoholism \\ 40 patients were alcoholic (26.6\%).}

\section{Clinical Profile/ Presenting Complaints}

Among the study group, 52 patients $(34.6 \%)$ had chest pain as initial presenting complaint and $34(22.6 \%)$ of patients presented with history of dyspnoea and $22(14.6 \%)$ presented with paroxysmal nocturnal dyspnoea, 45 (30\%) patients had focal neurological deficit at the time of presentation. 42 patients presented with hemiparesis and 3 patients presented with monoparesis. Sensory abnormalities were found only in one patient (Thalamic Haematoma). Eight patients presented with history of seizure (5.3\%) and 36 patients presented with history of LOC (24\%). Among the comorbidities, 22 patients had past history of Chronic Kidney Disease (14.6\%) and 22 patients had past history of Coronary Artery Disease (14.6\%). 
Blood Pressure Values

\begin{tabular}{|c|c|c|c|c|c|}
\hline & N & Minimum & Maximum & Mean & $\begin{array}{c}\text { Std. } \\
\text { Deviation }\end{array}$ \\
\hline SBP & 150 & 180 & 280 & 200.00 & 21.921 \\
\hline DBP & 150 & 120 & 160 & 124.80 & 6.525 \\
\hline Valid N & 150 & & & & \\
\hline \multicolumn{6}{|c|}{ Table 1. Blood Pressure Values } \\
\hline
\end{tabular}

Systolic Blood Pressure

51 patients had blood pressure in the range of $180-190$ $\mathrm{mmHg}(34 \%)$.

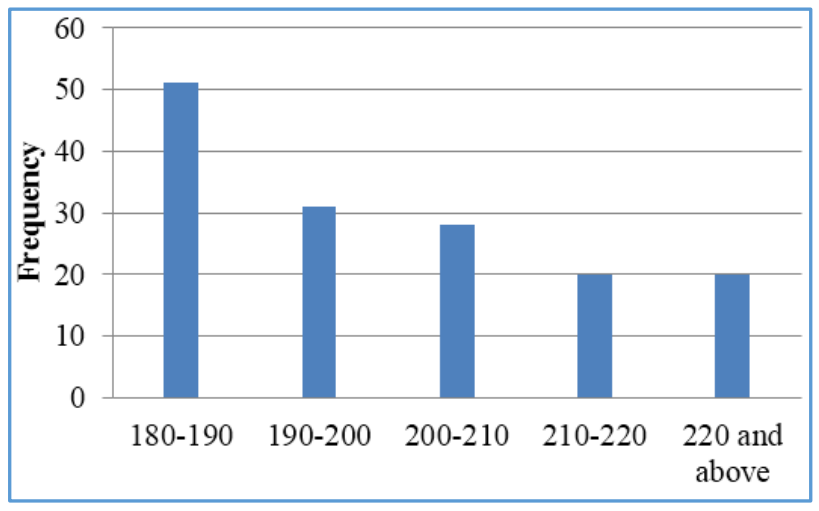

Figure 2. Systolic Blood Pressure

\section{Diastolic Blood Pressure}

Diastolic BP range in most of the patients was $120-130$ mmHg $(23.3 \%)$.

\section{Pulse Pressure}

Pulse pressure range in most of the patients was $50-60$ mmHg (30\%).

\section{Fundoscopy Findings}

All patients were subjected to fundoscopic examination. Fundoscopy findings of grade 3 and grade 4 had statistically significant association with elevation of systolic blood pressure. 21 patients with diastolic blood pressure $\geq 120$ mmHg had evidence of retinopathy on fundoscopy. 7 of them had grade 3 and 4 changes on fundoscopy.

\section{Laboratory Values}

Among the patients who underwent various investigations, 43 patients had microalbuminuria $(28.6 \%)$ and 110 patients had a creatinine value less than $2 \mathrm{mg} / \mathrm{dL}$ and serum creatinine value was more than $2 \mathrm{mg} / \mathrm{dL}$ in 40 patients. Severity of systolic blood pressure had a statistical significant association with serum creatinine elevation ( $p$ value- 0.00 ). Hyponatraemia was found in $28 \%$ and Hypokalaemia was found in $14 \%$ and Hyperkalaemia was seen in 10\%. Troponin I assay was done in patients with symptoms and ECG changes suggestive of acute coronary syndrome. Trop I positivity was found in 41 patients $(77 \%)$. Chest x-ray revealed cardiomegaly was found in around 12 patients and hilar prominence was found in 11 subjects and widened mediastinum was found in two subjects. ST/T changes were found in 49 patients $(32.6 \%)$. LVH voltage criteria was found in 16 patients $(10.6 \%)$.

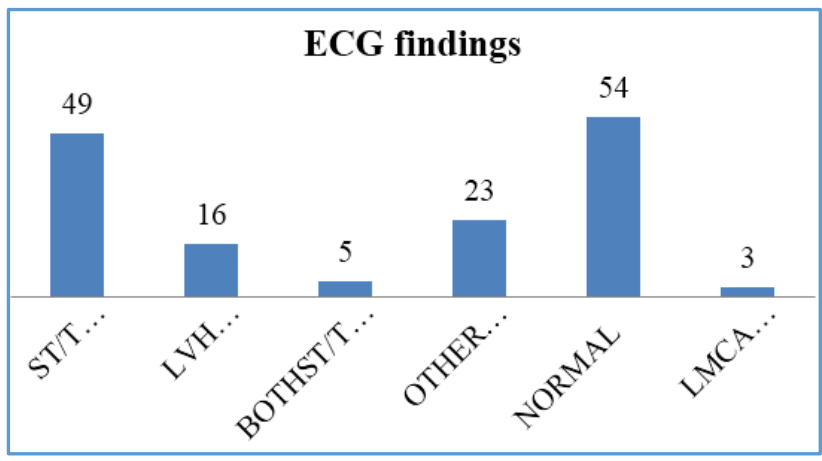

Figure 3. ECG Changes

\section{Renal Ultrasonogram}

Renal ultrasonogram was done in patients according to clinical criteria and laboratory values. 21 patients had grade 1 changes in ultrasonogram. 20 patients had grade 2 changes in ultrasonogram.

\section{Echocardiography}

23 patients had regional wall motion abnormality. 18 patients had left ventricular hypertrophy.

\section{CT Findings}

The most common finding as per imaging was intracerebral haemorrhage (52\%).

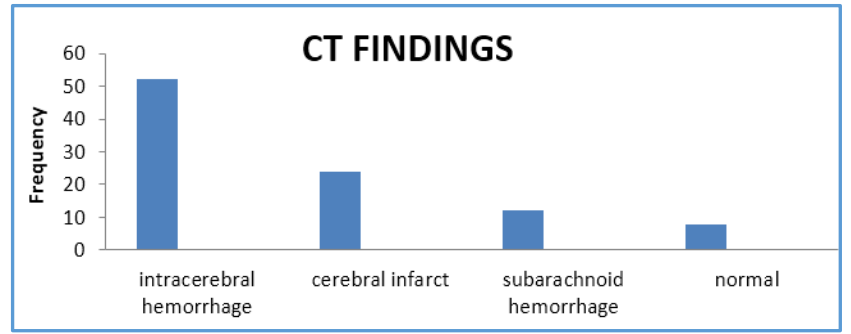

Figure 4. CT Head Findings

\section{Diagnosis}

The most common target organ damage found in the present study was acute coronary syndrome (35.3\%).

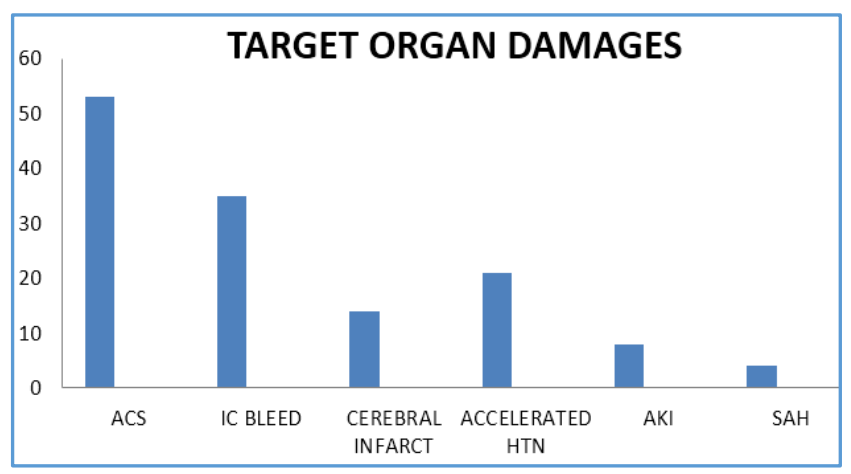

Figure 5. Target Organ Damages

\section{Target Organ Damages}

Acute Coronary Syndrome had a statistically significant association with severity of systolic blood pressure, diastolic blood pressure and pulse pressure. Cerebral infarct had a statistically significant association with severity of systolic blood pressure elevation as well as severity of elevation of 
diastolic blood pressure. Chronic kidney disease has a statistically significant association with severity of elevation of Systolic Blood Pressure and Diastolic Blood Pressure ( $p$ value- 0.00 ).

\section{Mortality Association and Statistical Significance Socio-Demographic Profile}

Age and mortality association was not statistically significant ( $p$ value- 0.134). 42 patients died in hospital, of which 35 were males and 7 were females. The association between sex and mortality was not found to be statistically significant ( $p$ value- 0.789 ).

\section{Aetiological Profile \\ Lifestyle}

Sedentary lifestyle is not statistically associated with mortality ( $p$ value- 0.49 ).

\section{Hypertensive Status}

109 patients were known hypertensives. Of the known hypertensives, 28 patients died $(25.6 \%)$. Patients with unknown status of hypertension has statistically significant high mortality rate (35\%) ( $p$ value- 0.49). Poor drug compliance has statistically significant association with mortality ( $p$ value- 0.00 ).

\section{Diabetes Mellitus}

Known diabetic status has a statistically significant association with mortality ( $\mathrm{p}$ value- 0.049 ).

\section{Addictions}

Smoking is not statistically significant in the context of mortality ( $p$ value- 0.075). Alcoholism was not having statistically significant association with mortality ( $\mathrm{p}$ value0.118).

\section{Co-Morbidities}

The past history of cerebrovascular accident had no statistical significance in terms of mortality ( $p$ value- 0.241 ). Past history of coronary artery disease was not having statistical significance in terms of mortality ( $p$ value- 0.267 ). 22 patients had history of chronic kidney disease. Of them 16 died (72\%). The presence of chronic kidney disease was found to be statistically significant in terms of mortality $(p$ value- 0.00 ).

\section{Clinical Profile}

\section{Presenting Complaints}

The focal neurological deficit had a statistically significant association with mortality, $(p=0.001)$. Mortality rate was high in those presented with seizure initially (7 patients$4.6 \%)$. Seizure is statistically significant in terms of mortality ( $\mathrm{p}$ value- 0.000$) .52$ patients $(34.6 \%)$ had chest pain at presentation, which has statistically significant association with mortality ( $p$ value- 0.00 ).

\section{Signs}

Patients whose initial systolic blood pressure more than $220 \mathrm{mg}$ has statistically significant mortality ( $\mathrm{p}$ value- 0.000 ).

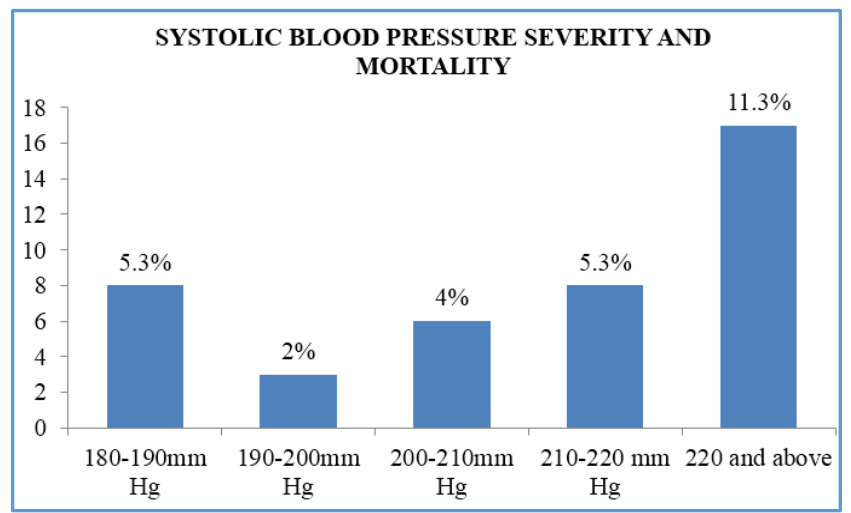

Figure 6. Systolic Blood Pressure Severity and association between Mortality

Patients with high Diastolic Blood Pressure (more than $150 \mathrm{mmHg}$ ) has statistically significant mortality ( $\mathrm{p}$ value$0.000)$.

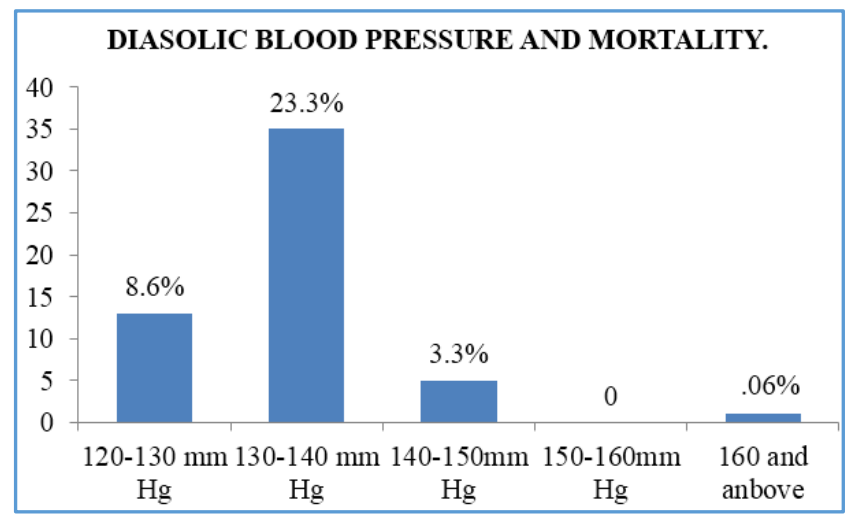

Figure 7. Diastolic Blood Pressure Severity and Mortality

Pulse pressure value of 110 and above (64\%) has statistically significant mortality ( $p$ value- 0.00 ).

Depressed sensorium was statistically significant in terms of mortality ( $p$ value- 0.000 ). The fundoscopic findings of higher grades (3 and 4) had a statistically significant mortality rate ( $p$ value- 0.000 ). Higher grades of motor weakness were statistically significant in terms of mortality ( $p$ value- 0.001 ).

\section{Routine Investigations}

Forty patients had a creatinine value more than $2 \mathrm{mg} / \mathrm{dL} ; 14$ died (35\%). High creatinine value (> $2 \mathrm{mg} / \mathrm{dL}$ ) was statistically significant in terms of mortality ( $p$ value- 0.00 ). Microalbuminuria had a statistically significant association with mortality ( $p$ value- 0.018 ). Trop I positivity was found to be statistically significant ( $p$ value- 0.001). ECG changes (ST/T changes) had statistically significant association with mortality ( $p$ value- 0.016 ). The presence of echo findings of regional wall motion abnormality is found to be statistically significant in terms of mortality ( $p$ value- 0.008). The ultrasonogram grading of grade 2 kidney damage is not found to be statistically significant in terms of mortality ( $\mathrm{p}$ value0.421).

\section{Diagnosis}

Intracerebral bleed was statistically significant in terms of mortality ( $p$ value- 0.000 ). Acute coronary syndrome was statistically significant in terms of mortality ( $p$ value- 0.000 ). 
Of all the diagnoses, intracerebral bleed had the highest mortality rate $(62.9 \%)$ followed by aortic dissection $(33.3 \%)$. Least mortality was associated with cerebral infarct (14.3\%).

\section{DISCUSSION \\ Age Distribution}

The frequency of end organ damage in the present study was the highest in 7 th decade (mean age $61.34+/-13.628$ ). The study done by Deshmukh et al on clinical profile of hypertensive emergencies ${ }^{4}$ states that the frequency of target organ damage was found to be highest in $7^{\text {th }}$ decade (mean age $67.79+/-8.23$ ). The age range in the present study was 26 to 90 years. With regards to the sex distribution the number of males presenting with hypertensive emergencies was more than the number of females, $74.6 \%$ were males. The study by Sunjay Gulhane et $\mathrm{al}^{5}$ and Martin et al ${ }^{6}$ both had a similar sex distribution. In the present study, on analysing age distribution gender wise, most of the males presenting with hypertensive emergencies were found to be mostly older than 50 years. The majority of female patients belonged to post-menopausal age group, which shows susceptibility of postmenopausal age group to organ damage.

\section{The Aetiological Profile Hypertensive Status}

Majority of the patients with hypertensive emergencies were known hypertensives (72\%). The categories of secondary hypertension found were renal parenchymal diseases (10\%), acute glomerulonephritis (.6\%) and renal artery stenosis (.6\%). Study by Martin et al ${ }^{6}$ identified $83 \%$ subjects as known hypertensives. In the present study, $23 \%$ of known hypertensives ignored their hypertensive status and discontinued hypertensive medications which might have worsened their target organ damage.

The major co-morbidities present in our study were diabetes mellitus and dyslipidaemia. Diabetes was present in $36 \%$ of patients and dyslipidaemia was found in $27.3 \%$ of patients and $25 \%$ patients had dyslipidaemia. In the study done by Martin et al, $626 \%$ of patients had diabetes mellitus. With more studies coming in, the aetiologic link between insulin resistance and arterial hypertension is becoming more evident. Sanjay et al ${ }^{5}$ found that severity of systolic blood pressure elevation had statistically significant association with diabetes and dyslipidaemia.

\section{Lifestyle}

Sedentary lifestyle was found in $47 \%$ of patients. Jose et al 7 in their study showed that sedentary lifestyle was present in $50 \%$ of patients. In our context, the patients were coming from both urban and rural areas. Patients coming from urban areas were found to have less physical activity.

\section{Clinical Profile}

Analysing the present data, the presenting complaints related to various target organ damages were chest pain (34.6\%) followed by focal neurological deficit (30\%), loss of consciousness $(24 \%)$ and dyspnoea (22\%). Guilano et al ${ }^{8}$ in his study found chest pain in $28.3 \%$ followed by focal neurological deficit (24\%).

The highest recorded systolic blood pressure was 280 mmHg with mean systolic blood pressure of
$200 \pm 21.921 \mathrm{mmHg}$. Highest diastolic blood pressure was found to be $160 \mathrm{mmHg}$ with a mean of $124.8 \pm 6.525 \mathrm{mmHg}$. Sanjay et $\mathrm{al}^{6}$ in his study found that mean systolic blood pressure was $208 \pm 39 \mathrm{mmHg}$ and mean diastolic blood pressure was $140 \pm 18.5 \mathrm{mmHg}$. Jose et al7 found the mean systolic blood pressure to be $192 \mathrm{mmHg}$ with a standard deviation of $26 \mathrm{mmHg}$ and mean diastolic blood pressure of $129 \mathrm{mmHg}$ with a standard deviation of $12 \mathrm{mmHg}$ respectively.

In our study, fundoscopy findings were as follows. 31 patients showed grade 1 changes; normal in 47 subjects (31\%). Papilloedema was present in 40 patients $(20 \%)$. Sanjay et $\mathrm{al}^{5}$ found that fundoscopic evaluation was normal in $30 \%$ of subjects followed by grade 1 changes (20\%), grade 2 changes (24\%), grade 3 changes (13\%) and papilloedema in $4 \%$.

\section{Laboratory Findings and Investigations}

In the present study, microalbuminuria was found in $29.8 \%$ of patients. Microalbuminuria was found in $28.6 \%$ of patients in the study done by Bal K Sharma ${ }^{9}$ et al. In our study, it was found that $26 \%$ of patients had serum creatinine value more than $2 \mathrm{mg} / \mathrm{dL}$. Sanjay et $\mathrm{al}^{5}$ in their study found that $28 \%$ of patients having serum creatinine more than $2 \mathrm{mg} / \mathrm{dL}$. In the present study, $14 \%$ of patients had hypokalaemia compared to $10 \%$ with hyperkalaemia, reflecting secondary aldosteronism from increased renin secretion induced by intrarenal ischaemia. Computed Tomography findings showed that intracerebral haemorrhage was the commonest cause for neurological damage followed by cerebral infarct. In the present study, echocardiography shows regional wall motion abnormalities in 23 patients (43.3\%) and left ventricular hypertrophy in 18 patients $(33.3 \%)$. Shapiro et al ${ }^{10}$ study showed similar findings.

\section{Target Organ Damage}

Evaluation of target organ damage showed that acute coronary syndrome was the commonest type (35\%) followed by cerebrovascular accident-intracerebral bleed $(23.3 \%)$ and acute ischaemic stroke $(9.3 \%)$. Jose et al ${ }^{7}$ found acute coronary syndrome in $25.1 \%$ followed by ischaemic stroke in $22.9 \%$ and haemorrhagic stroke in $14.8 \%$. Fernando et al in their study showed that target organ damage related to cardiovascular system was $59 \%$ followed by CNS related damages. The difference may be due to selection bias.

\section{Mortality Profile}

The present study shows that age has no statistically significant relation with hypertensive emergency. Bal $\mathrm{K}$ Sharma et al found that age had a statistically significant correlation with mortality in hypertensive emergencies. Fernando et al in their study showed that age had a statistically significant correlation with mortality ( $p$ value0.001). The present study showed that sex does not have a statistically significant relation with mortality. According to Bal K Sharma et al, sex does not have a statistically significant correlation with mortality.

\section{Co-Morbidities and Mortality Status}

The present study showed that past history of cerebrovascular disease had no statistical significant relation with mortality. The present study showed that past history of 
coronary artery disease had no statistical significance in terms of mortality. James et al found that history of heart failure had a statistical significant relation with mortality. James et al found that history of cerebrovascular accident had a statistical significance in relation with mortality. The difference may be due to selection bias. The present study showed that there was a statistically significant relation between chronic kidney disease and mortality ( $p$ value0.001 ). Jose et al in their study showed a similar relation between chronic kidney disease and mortality.

\section{Mortality Profile}

\section{Aetiological Profile and Mortality Status}

Fernando et al showed that irregular hypertensive treatment was statistically significant in terms of mortality. According to Framingham heart study, monitored treatment for hypertension was associated with low incidence of hypertensive emergencies. Jose et al found that irregular antihypertensive medication had statistical significant association with mortality.

\section{Risk Factors and Mortality Status}

In our study, it was found that diabetes was associated with mortality ( $p$ value $<0.05$ ). This fact was comparable to the study done by Rasheed et al, whose study showed that diabetics had a more mortality rate and this relation was found to be statistically significant. Saguner et al in their study ${ }^{11}$ showed that non-adherence to medication had statistically significant association with mortality.

\section{Clinical Profile and Mortality Status}

The present study showed that chest pain at presentation had a statistical significance with mortality. Jose et al found that presence of chest pain had a statistically significant relation with mortality. The present study showed that dyspnoea at presentation had no statistically significant relation with mortality. Bal K Sharma also found that dyspnoea had no statistical significance in terms of mortality. The present study showed that focal neurological deficit at presentation had a statistically significant association with mortality. Fernando et al found that neurological deficit had a statistical significance in association with mortality (0.001). Jose et al found that presence of focal neurological deficit was statistically significant in terms of mortality.

The present study showed that loss of consciousness at presentation had a statistically significant association with mortality ( $p$ value- 0.000 ). Fernando et al found that loss of consciousness as initial presenting complaint had a statistical significance. Jose et al found that presence of loss of consciousness had statistically significant association with mortality. The present study showed that depressed sensorium had a statistically significant association with mortality. Gulhane et al found that depressed sensorium had a statistical significant association with mortality.

\section{Clinical Signs and Mortality Status}

The present study showed that severity of systolic blood pressure elevation had a statistically significant association with mortality. Diallo et al ${ }^{12}$ found that severity of systolic blood pressure had statistical significance in terms of mortality ( $p$ value 0.001 ). The present study showed that severity of elevation of Diastolic Blood Pressure had a statistically significant relation with mortality. Diallo et al found that diastolic blood pressure elevation had association with mortality ( $p$ value- 0.02 ). The present study showed that pulse pressure elevation was significantly associated with mortality. Fernando et al in their study showed that pulse pressure had statistically significant association with mortality. The present study showed that grade $3(20 \%)$ and grade 4 retinopathy (26\%) were statistically associated with mortality. According to the study of Bal K Sharma et al, death in grade 3 retinopathy was $14 \%$ and death in grade 4 retinopathy was $18.2 \%$.

A study done by Lip GY et al ${ }^{13}$ on complications and survival of 315 patients with malignant hypertension found a low median survival time in patients with proteinuria and high serum urea and creatinine at presentation. Microalbuminuria had statistical significance in terms of mortality in the present study ( $p$ value- 0.018 ). Messerli et al showed that proteinuria is a statistically significant predictor of mortality.

The present study showed that elevation of serum creatinine more than $2 \mathrm{mg} / \mathrm{dL}$ had an association with mortality. Bal K Sharma et al in their study found that serum creatinine value $>2 \mathrm{mg} / \mathrm{dL}$ was significant in terms of mortality. Lip GY et al found that patients presented with accelerated hypertension, whose serum creatinine level more than $2 \mathrm{mg} / \mathrm{dL}$ had a worse survival and this was statistically significant. Lip GY et al showed that percentage of patients with acute severe hypertension having chronic kidney disease was $31.7 \%$ on admission. Lynda et al showed that among hypertensive emergency patients, alteration of kidney function was statistically associated with mortality.

Echocardiographic findings of regional wall motion abnormality and left ventricular hypertrophy had a statistical significance in terms of mortality ( $p$ value- 0.008 ). In the study by Bal K Sharma et al, it was found that regional wall motion abnormality was present in $52.3 \%$ of patients and was associated with increased mortality. The study of Bal K Sharma also found that left ventricular hypertrophy had a statistical significant association with mortality.

The present study about hypertensive emergency showed that patients with acute stroke as target organ damage had a statistically significant association with mortality. Diallo et al showed that stroke had high mortality (25.4\%) and had a statistical significance with mortality. The present study showed that in hospital mortality of hypertensive emergency was $28 \%$ and had statistically significant association with mortality. Patne et $\mathrm{al}^{14}$ found that high blood pressure at the time of presentation had adverse outcome and was statistically significant.

\section{CONCLUSION}

1. Majority of patients presenting with hypertensive emergency belongs to sixth decade of life.

2. Males have a higher predilection to have hypertensive emergencies compared to females.

3. The most common aetiology associated with hypertensive emergencies was essential hypertension.

4. The in-hospital mortality of these patients was found to be $28 \%$. 
5. Presence of chest pain, seizures and loss of consciousness had a significant higher rate of mortality rate in patients with hypertensive emergency.

6. Higher values of systolic blood pressure, diastolic blood pressure and pulse pressure were associated with higher mortality rate.

7. Presence of microalbuminuria was a poor prognosticator factor.

8. Depression of sensorium at presentation is significant in terms of mortality.

9. Acute coronary syndrome was the most common target organ damage followed by intracerebral haemorrhage.

10. Ischaemic changes in ECG and regional wall motion abnormality in echocardiogram were very well correlating with higher mortality in patients with hypertensive emergency.

\section{REFERENCES}

[1] Rosen CJ. Clinical practice. Vitamin D insufficiency. N Engl J Med 2011;364(3):248-54.

[2] Zampaglione B, Pascale C, Marchisio $M$, et al. Hypertensive urgencies and emergencies. Prevalence and clinical presentation. Hypertension 1996;27(1):144-7.

[3] Chobanian AV, Bakris GL, Black HR, et al. The seventh report of the Joint National Committee on Prevention, Detection, Evaluation and Treatment of High Blood Pressure: the JNC 7 report. JAMA 2003;289(19):256072.

[4] Deshmukh A, Kumar G, Kumar N, et al. Effect of Joint National Committee VII report on hospitalizations for hypertensive emergencies in the United States. Am J Cardiol 2011;108(9):1277-82.

[5] Gulhane S, Chopade B, Sundar U, et al. Study of clinical profile of patients with hypertensive urgencies and emergencies. IOSR Journal of Dental and Medical Sciences 2016;15(6):24-9.
[6] Martin JFV, Higashiama E, Garcia E, et al. Hypertensive crisis profile. Prevalence and clinical presentation. Arquivos Brasileiros de Cardiologia 2004;83(2):131-6, 125-30.

[7] Vilela-Martin JF, Vaz-de-Melo RO, Kuniyoshi CH, et al. Hypertensive crisis: clinical-epidemiological profile. Hypertension Research 2011;34(3):367-71.

[8] Pinna G, Pascale C, Fornengo P, et al. Hospital admissions for hypertensive crisis in the emergency departments: a large multicentre Italian study. Plos One 2014;9(4):e93542.

[9] Sharma BK, Singh G, Sagar S. Malignant hypertension in north-west India. A hospital based study. Jpn Heart J 1994;35(5):601-9.

[10] Shaprio LM, Beevers DG. Malignant HTN: cardiac structure and function at presentation and during therapy. Br Heart J 1983;49(5):477-84.

[11] Saguner AM, Dur S, Perrig M, et al. Risk factors promoting hypertensive crisis: evidence from a longitudinal study. American Journal of Hypertension 2010;23(7):775-80.

[12] Diallo AD, Mouanodji MB, Niamkey EK, et al. Clinical features of the arterial hypertension in a medical emergency service in Abidjan. African Journal of Nephrology 1999;3:84-7.

[13] Lip GY, Beevers M, Beevers DG. Complications and Survival of 315 patients with malignant-phase hypertension. J Hypertens 1995;13(8):915-24.

[14] Patne SV, Chintale KN, Tungikar S, et al. Clinical study of hypertensive emergencies in young patients in tertiary health care centre. Asian Pacific Journal of Health Sciences 2016;3(1):7-15. 